\title{
Spontaneous Neurological Deterioration in Acute Cardioembolic Stroke: A Subgroup of Patients With Early Severe Prognosis
}

\author{
Adria Arboix ${ }^{\mathrm{a}, \mathrm{c}}$, Adela Vicens ${ }^{\mathrm{a}}$, Josep Maria Vives ${ }^{\mathrm{a}}$, \\ Luis Garcia-Eroles ${ }^{\mathrm{b}}$, Joan Massons ${ }^{\mathrm{a}}$
}

\begin{abstract}
Background: To determine clinical factors of spontaneous early neurological deterioration (END) and prognosis in a cohort of 480 consecutive patients with cardioembolic stroke (CS) without thrombolytic therapy hospitalized within 24 hours of the onset of symptoms.
\end{abstract}

Methods: END was defined as a drop of $\geq 1$ point in the Canadian Stroke Scale or $\geq 2$ points in the Glasgow Coma Scale between admission and after 72 hours. All patients underwent computerized tomography and/or MRI examination.

Results: END is present in $8.3 \%$ of CS patients. CS patients with END $(n=40)$ in comparison with patients without ND $(n=440)$ showed a worse early prognosis with statistically significant differences in absence of neurological deficit at hospital discharge $(5 \%$ versus $17.3 \%$ ), length of hospitalization (30.8 versus 18.5 days) and in-hospital mortality $(47.5 \%$ versus $8.4 \%)$. In the multivariate analysis, early seizures, severe headache and hypertension were independent clinical predictors of END.

Conclusions: In patients with CS, early seizures, severe headache and hypertension are clinical variables associated with END. CS with END constitutes a subgroup of patients with severe prognosis. Because most causes of worsening can be treated effectively, the

Manuscript accepted for publication October 17, 2011

${ }^{\mathrm{a}}$ Cerebrovascular Division, Department of Neurology, University of Barcelona, Hospital Universitari del Sagrat Cor, Barcelona, Spain ${ }^{\mathrm{b}}$ Clinical Information Systems, Consorci Sanitari del Maresme, Mataro, Barcelona, Spain

${ }^{\mathrm{c} C o r r e s p o n d i n g ~ a u t h o r: ~ A d r i a ~ A r b o i x, ~ C e r e b r o v a s c u l a r ~ D i v i s i o n, ~}$ Department of Neurology, Hospital Universitari del Sagrat Cor, Viladomat 288, E-08029 Barcelona, Spain. Email: aarboix@hscor.com

doi: $10.4021 / \mathrm{jnr} 62 \mathrm{w}$ deteriorating cardioembolic stroke patient merits a swift and incisive diagnostic and therapeutic approach.

Keywords: Cerebrovascular disease; Headache; Cardioembolic stroke; Early neurological deterioration; Early seizures; Hypertension; Prognosis

\section{Introduction}

Cardioembolic ischemic stroke accounts for one quarter of all cerebral infarcts [1]. Cardioembolic ischemic stroke is the most severe ischemic stroke subtype, with a low frequency of absence of neurological dysfunction at hospital discharge and a non-negligible risk of early embolic recurrence (1$10 \%$ ) [1-4]. Cardioembolic stroke is also the stroke subtype with the highest in-hospital mortality (6-27\%) [1, 2-4]. Cardioembolic stroke is a homogeneous subtype of ischaemic stroke characterized by sudden onset of neurological deficit [1]. Patients with acute ischemic stroke of cardioembolic origin show maximal neurological at stroke onset or within a few minutes after the onset of symptoms, which is contrast with patients suffering from atherothrombotic stroke or lacunar infarction (LI) in which neurological dysfunction is progressively established in the course of several hours. Progression was most frequent in patients with LI (37\%) and large-artery occlusive disease (33\%) and least frequent in patients with embolism (7\%) [5].

Spontaneous early neurological deterioration (END) has been reported in 12 to $43 \%$ of patients with acute ischaemic stroke and is associated with an unfavourable outcome, with increased morbidity and mortality [6-9]. However, there is little data on END when patients are individualized by the different subtypes of ischaemic stroke. Therefore, progressing or deteriorating stroke is uncommon in cardioembolic stroke and clinical factors and prognosis associated with early neurological deterioration in this stroke subtype have been scarcely assessed. The objective of this study was to identify prognosis and clinical variables associated with cardioembolic stroke patients associated with spontaneous early neurological deterioration. 


\section{Materials and Methods}

Since January 1986 an ongoing hospital-based stroke registry has been established at the Hospital of Sagrat Cor (an acute-care 350-bed teaching hospital in the city of Barcelona), so that data of all first-ever stroke patients admitted consecutively to the Department of Neurology of the hospital are collected prospectively in the stroke registry [10]. Data from all patients are entered following a standardized protocol with 186 items regarding demographic features, risk factors, clinical findings, laboratory and neuroimaging data, complications, and outcome. The use of the same protocol for all patients ensures completeness of the information in the database. Subtypes of stroke were classified according to the Cerebrovascular Study Group of the Spanish Society of Neurology [11], which is similar to the National Institute of Neurological Disorders and Stroke Classification [12]. Definitions of cardiovascular risk factors and stroke subtype were those used by our group in previous studies $[10,11$, 13].

For the purpose of this hospital-based prospective study, the cohort of 669 patients with cardioembolic infarction included in the stroke registry up to December 2002, the particular time at which data of 3420 patients had been entered in the database was selected. The remaining patients with transient ischaemic attack (TIA), ischaemic stroke (atherothrombotic infarction, lacunar infarction, infarction of unusual cause, infarction of undetermined cause), intracerebral haemorrhage, subarachnoid haemorrhage, and spontaneous subdural hematoma were excluded.

As in previous studies, to classify a patient as having cardioembolic infarction $[10,13,14]$ the following was required: presence of a medium-sized (maximal diameter of the lesion, 1.5 to $3 \mathrm{~cm})$ or large $(>3 \mathrm{~cm})$ cerebral infarction; stroke onset of non-lacunar syndrome during ordinary daily activities; duration of neurological deficit $>24$ hours; and identification of a commonly accepted cardiac source of embolus in the absence of stenosis $>50 \%$ in the ipsilateral supra-aortic trunks.

On admission, demographic characteristics, salient features of clinical history and neurologic examination, results of routine laboratory tests, chest radiography, and twelvelead electrocardiography were recorded. In all patients, brain CT scans were performed within the first week of hospital admission. Patients with negative results had a second CT during their stay in the hospital or were studied by MRI. Other investigations included angio-MRI (37.2\% of patients), Doppler ultrasonography of the supra-aortic trunks (41.1\%), arterial digital subtraction angiography $(4.5 \%)$, B-mode transthoracic or transesophageal echocardiography $(27.7 \%)$, immunological testing (8.1\%) and lumbar puncture (1\%).

Stroke severity was quantified by an experienced neurologist using the Canadian Stroke Scale (total score from 1.5 $=$ maximal deficit to $10=$ absence of deficit) [15]. In agree- ment with previous studies [9], spontaneous early neurological deterioration was defined as a drop of $\geq 1$ point in the Canadian Stroke Scale or $\geq 2$ points in the Glasgow Coma Scale between admission and after 72 hours. Patients with fluctuating symptoms not showing decline in the Canadian Stroke Scale or Glasgow Coma Scales at 72 hours were not diagnosed as having early neurological deterioration. The modified Rankin scale (mRS) was used to evaluate clinical outcome at hospital discharge [16].

Demographic variables included age and sex. All other findings were dichotomized as present versus absent. Anamnestic findings consisted of history of hypertension, diabetes, myocardial infarction or angina, rheumatic heart disease, congestive heart failure, atrial fibrillation, heavy smoking ( $>20$ cigarettes/day), alcohol abuse ( $>80 \mathrm{~g} /$ day), intermittent claudication, TIA, previous cerebral infarction, hyperlipidemia, nephropathy, cirrhosis or chronic liver disease, chronic obstructive pulmonary disease (COPD), and age of 85 years or older. Clinical variables included sudden onset of symptoms (minutes), acute onset (hours) and subacute onset (days); headache; dizziness; early seizures; nausea or vomiting; altered consciousness (drowsy, stuporous, comatose); limb weakness (hemiparesis or hemiplegia, Babinski's sign not mandatory); sensory symptoms; hemianopia; aphasia or dysarthria; ataxia; and cranial nerve palsy. Neuroimaging variables included middle cerebral artery involvement, cerebral anterior topography, cerebral posterior topography, posteroinferior cerebellar artery topography, arterial basilar topography, occipital involvement, cerebellum and other topographies.

Cardioembolic stroke-related headache was defined as severe headache (incapacitating in intensity spontaneously referred by the patient and requiring analgesic treatment, usually paracetamol) at the time of stroke onset and assessed by a neurologist or a physician in the emergency department. Headache type (tension-type, migraine, other) was defined according to criteria of the Headache Classification Committee of the International Headache Society [17] and concomitant nausea or vomiting. This information was obtained on admission to the hospital. Patients in whom headache was not recorded due to communication difficulties, including acute confusional state, aphasic disorder or a low level of consciousness (Glasgow Coma Scale < 14) [18] in whom no detailed information about headache at stroke onset could not be obtained were excluded from the study. In all patients with severe headache work-up studies were performed in order to exclude the concomitant presence of subarachnoid haemorrhage, arterial dissection, central venous thrombosis or Horton's temporal (giant cell) arteritis.

Early epileptic seizures were defined as those starting at the beginning or within 48 hours of the cerebrovascular event in a patient without history of seizures. Limb-shaking TIA, transient hemiballism/hemichorea, decerebrate fits and convulsive-like movements (in brainstem stroke) were ex- 
Table 1. Clinical Differences in Patients With Cardioembolic Stroke According to Presence or Absence of Early Neurological Deterioration

\begin{tabular}{|c|c|c|c|}
\hline \multirow{2}{*}{ Data } & \multicolumn{2}{|c|}{ Early neurological deterioration } & \multirow{2}{*}{ P value } \\
\hline & Absent, $n=440$ & Present, $n=40$ & \\
\hline Male sex & $163(37)$ & $14(35)$ & 0.471 \\
\hline Age, years, mean (SD) & $77.6(9.7)$ & $76.4(9.3)$ & 0.891 \\
\hline \multicolumn{4}{|l|}{ Vascular risk factors } \\
\hline Atrial fibrillation & $319(72.5)$ & $29(72.5)$ & 0.564 \\
\hline Hypertension & $214(48.6)$ & $27(67.5)$ & 0.016 \\
\hline Diabetes mellitus & $79(18)$ & $9(22.5)$ & 0.300 \\
\hline Ischaemic heart disease & $96(21.8)$ & $7(17.5)$ & 0.342 \\
\hline Valvular heart disease & $95(21.6)$ & $7(17.5)$ & 0.354 \\
\hline Congestive heart failure & $40(9.1)$ & $4(10)$ & 0.511 \\
\hline Previous TIA & $44(10)$ & $4(10)$ & 0.630 \\
\hline Obesity & $7(1.6)$ & $3(7.5)$ & 0.043 \\
\hline \multicolumn{4}{|l|}{ Clinical features } \\
\hline Sudden onset & $324(73.6)$ & $25(62.5)$ & 0.094 \\
\hline Severe headache & $31(7)$ & $7(17.5)$ & 0.029 \\
\hline Very early seizures & $6(1.4)$ & $3(7.5)$ & 0.032 \\
\hline Limb weakness & $336(76.4)$ & $31(77.5)$ & 0.524 \\
\hline Sensory symptoms & $156(35.5)$ & $17(42.5)$ & 0.235 \\
\hline Hemianopia & $84(19.1)$ & $9(22.5)$ & 0.365 \\
\hline Speech disturbances & $304(69.1)$ & $29(72.5)$ & 0.401 \\
\hline Nausea, vomiting & $25(5.7)$ & $3(7.5)$ & 0.419 \\
\hline Ataxia & $23(5.2)$ & $1(2.5)$ & 0.388 \\
\hline Cranial nerve palsy & $13(3)$ & $1(2.5)$ & 0.672 \\
\hline In-hospital mortality & $37(8.4)$ & $19(47.5)$ & 0.000 \\
\hline Symptom-free at discharge & $76(17.3)$ & $2(5)$ & 0.04 \\
\hline Hospital stay, days, mean (SD) & $18.5(12.4)$ & $30.8(22.8)$ & 0.003 \\
\hline
\end{tabular}

cluded. Seizures were classified following the recommendation of the International League Against Epilepsy [19].

In the acute phase, the stroke patients were managed in accordance with recommendations of the Cerebrovascular Study Group of the Spanish Society of Neurology [20]. Main strategies were as follows: (a) maintenance of blood pressure without the use of hypotensive drugs unless the systolic blood pressure was $\geq 220 \mathrm{~mm} \mathrm{Hg}$ or the diastolic blood pressure $\geq 120 \mathrm{~mm} \mathrm{Hg}$; (b) early treatment of hyperglycemia avoiding the use of glucose infusion; (c) prevention of pulmonary thromboembolism with low-dose heparin; (d) early anticoagulation therapy except when antiplatelet was recommended, and (e) physical and respiratory therapy was performed during the patient's stay in hospital. No patient received thrombolytic therapy because such treatment was not allowed in our hospital until 2006.

Prior to conducting the study, approval was obtained from the Ethical Committee on Clinical Research of the hospital.

\section{Statistical analysis}

Demographic characteristics, clinical events, and outcome of patients with cardioembolic infarction with END were compared with patients without END. In the univariate analyses, continuous variables were analyzed with the Student's t-test and categorical variables with the chi-square $\left(\chi^{2}\right)$ test (with Yate's correction when necessary). Statistical significance was set at $\mathrm{P}<0.05$. Variables related to cardioembolic stroke with END in the univariate analysis plus age and sex were studied in a multiple linear regression model. Age was used as a continuous variable with a constant odds ratio (OR) for each year. The predictive model was based on demographic, vascular risk factors and clinical data. Cardioembolic infarc- 
Table 2. Independent Predictive Value of Different Variables Associated With Early Neurological Deterioration in Acute Cardioembolic Infarction

\begin{tabular}{lllll}
\hline Variable & $\boldsymbol{\beta}$ & SE $(\boldsymbol{\beta})$ & Odds ratio $(\mathbf{9 5 \%} \mathbf{C I})$ & P value \\
\hline Early seizures & 1.738 & 0.741 & $5.69(1.33-24.29)$ & 0.019 \\
Severe headache & 1.081 & 0.463 & $2.95(1.19-7.30)$ & 0.020 \\
Hypertension & 0.715 & 0.356 & $2.04(1.02-4.11)$ & 0.044 \\
\hline
\end{tabular}

$\beta=-3.004$, SE $(\beta)=0.297$, goodness-of-fit $\chi^{2}=0.024$, d.f. $=1, P=0.876$. Area under the ROC curve $=0.649$, sensitivity $75 \%$, specificity $48 \%$, positive predictive value $12 \%$, negative predictive value $95 \%$, correct classification $50 \%$.

tion with END, coded as absence $=0$, presence $=1$, was the dependent variable. The level of significance was set as 0.15 , and the tolerance level at 0.0001 . The maximum likelihood approach was used to estimate weights of the logistic parameters. Odds ratio and $95 \%$ confidence interval (CI) were calculated from the beta coefficients and standard errors. The hypothesis that the logistic model adequately fitted the data was tested by means of the goodness of fit $\chi^{2}$ test. The SPSS-PC+ and the BMDP computer programmes were used for statistical analyses.

\section{Results}

Of 669 patients with acute cardioembolic infarction collected from the stroke registry, 229 patients with communication difficulties including acute confusional state, aphasic disorder, or low level of consciousness were excluded. Of the remaining 480 patients with cardioembolic infarction, $40(8.3 \%)$ presented END. Thirty-five percent of patients were males, with a mean (SD) age of 76.4 (9.3) years. The most common vascular risk factors were atrial fibrillation in $72.5 \%$ of cases, arterial hypertension in $67.5 \%$ and diabetes in $22.5 \%$. Obesity was observed in $7.5 \%$ of cases. The most frequent clinical manifestations were limb weakness in $77.5 \%$ of cases, speech disturbances in $72.5 \%$, sensory symptoms in $42.5 \%$ and hemianopia in $22.5 \%$. A total of 7 patients $(17.5 \%)$ complained of severe, incapacitating headache which appeared simultaneously at the onset of the neurological deficit. Early seizures at stroke onset were observed in $7.5 \%$ of patients (generalized tonic-clonic without obvious focal onset).

Table 1 shows as comparison of risk factors, clinical data and outcome between the groups of patients with cardioembolic stroke with and without END. Hypertension, obesity, severe headache and in-hospital mortality were significantly more frequent and symptom free at discharge was less frequent in patients with END than in patients without END. After multivariate analysis, early seizures $(\mathrm{OR}=5.69)$, severe headache $(\mathrm{OR}=2.95)$ and hypertension $(\mathrm{OR}=2.04)$ were selected as independent predictors of END (Table 2).

\section{Discussion}

Cardioembolic stroke is a subtype of cerebral infarction in which maximal neurological deficit usually occurs suddenly at the onset of stroke in contrast to atherothrombotic stroke, lacunar infarction or infarction of unknown cause where neurological deficit generally progresses over the first hours after stroke onset $[2,21]$. In the present series, END was observed in $8.3 \%$ of patients. In accordance with data from patients with cerebral infarction in general showing that early neurological worsening is associated with poor prognosis, our patients with END compared with the group of patients without END showed higher in-hospital mortality rate $(47.5 \%$ versus $8.4 \%)$, lower percentage of symptom-free patients at hospital discharge (5\% versus $17.3 \%)$ and longer hospital stay (30.8 versus 18.5 days).

Many different causes are likely to contribute to the progressive deterioration of ischaemic stroke: 1) neurologic deterioration (gradual or stepwise progression of neurologic focal deficits while the patient usually remains alert and free of medical complications); 2) brain edema (a complication of mostly large strokes, especially hemorrhages that are accompanied by decreased consciousness); and 3) systemic medical complications, especially infection and fever which may also lead to increased brain ischemia. Other medical complications include, high serum glucose levels, changes in blood pressure, thrombus propagation, seizures, low collateral blood flow, cerebral hemodynamic reserve impairment and neuroexcitotocity [5-8, 22, 23].

Interestingly, the multivariate analysis showed in our study the presence of three independent clinical variables associated with END, including early seizures $(\mathrm{OR}=5.69)$, severe headache $(O R=2.95)$ and hypertension $(O R=2.04)$ Early epileptiform activity has a deleterious effect on infarcted areas, perhaps by an anoxic event, and contributes to secondary brain damage, which may favour the presence of END. Early seizures occurred in the temporal window where a penumbra of potentially salvageable brain tissue is believed to exist and this may determine a greater area of definite cerebral infarction, which in turn would be associated with a poorer clinical course [24-26]. The SASS inves- 
tigators found higher mortality rates among stroke patients with seizures after 30 days and 1 year [27]. Those with postischaemic seizures also had a significantly poorer neurological score during the acute hospitalization and worse Rankin scores at follow-up (median 9 months). These data would coincide, in part, with the present findings in which the poorer outcome of patients with early seizures would be mediated by a higher probability of END.

The presence of severe headache at the onset of cardioembolic stroke occurred in $17.5 \%$ of patients with END compared with $7 \%$ in patient with non-END $(\mathrm{P}=0.029)$. Moreover, severe headache was an early clinical marker significantly associated with END in the logistic regression analysis $(\mathrm{OR}=2.95)$. In a previous study by our group [28], the incidence of headache in cardioembolic stroke was 39\% (severe-incapacitating $11 \%$ and mild-moderate intensity in $28 \%$ ), which is similar to the frequency reported in other studies [29, 30]. As far as we are aware, this is the first study showing an association between severe headache at stroke onset and END in patients with cardioembolic infarction.

The association between headache and neurologic impairment may be in part the result of the effect of brain edema and/or the effect of excitatory neurotransmitters. Leira et al [30] suggested that headache at stroke onset of ischaemic stroke is an independent predictor of neurological worsening and hypothesized that headache might be a surrogate marker of the molecular mechanisms involved in neurological worsening after acute stroke. CSF concentrations of glutamate, interleukin- 6 and nitric oxide metabolites were significantly greater in patients with progressive stroke and these biochemical markers were also significantly higher in patients with headache than in those without headache, as well as associated with the presence of early signs of cerebral ischaemia in brain CT studies [23, 30, 31].

On the other hand, and in agreement with other studies $[8,23]$, history of arterial hypertension is related to END. Severe hypertension may promote early brain edema and increased risk of haemorrhagic transformation $[8,9]$.

The characteristics of the patients included in the present study are not influenced by thrombolityc treatment [32] because thrombolysis was introduced as standard treatment in our hospital in 2006. Our study therefore allows a better understanding of the natural history of a subgroup of patients with cardioembolic stroke and severe early outcome.

In summary, cardioembolic stroke with END is a subgroup of patients with high early death and severe prognosis. Early seizures, severe or incapacitating headache at the onset of cardioembolic stroke and arterial hypertension adversely affects outcome in acute cardioembolic ischemic stroke and are clinical factors associated with END. Because most causes of worsening can be treated effectively, the predictive clinical factors of deteriorating cardioembolic stroke merits a swift and incisive diagnostic and therapeutic approach.

\section{Acknowledgements}

We thank Drs Noemi Amoros, Michael Lowak and Anna Cartanya for their valuable collaboration in the collection of data from the stroke registry, and Dr Marta Pulido for editing the manuscript and editorial assistance.

\section{Conflict of Interest}

No conflict of interest.

\section{Sources of Funding}

This study was supported in part by a grant from Fondo de Investigaciones Sanitarias (FIS PI/081514), Instituto de Investigacion Carlos III, Madrid, Spain.

\section{References}

1. Arboix A, Alio J. Cardioembolic stroke: clinical features, specific cardiac disorders and prognosis. Curr Cardiol Rev. 2010;6(3):150-161.

2. Ferro JM. Cardioembolic stroke: an update. Lancet Neurol. 2003;2(3):177-188.

3. MacDougall NJ, Amarasinghe S, Muir KW. Secondary prevention of stroke. Expert Rev Cardiovasc Ther. 2009;7(9):1103-1115.

4. Khoo CW, Lip GY. Clinical outcomes of acute stroke patients with atrial fibrillation. Expert Rev Cardiovasc Ther. 2009;7(4):371-374.

5. Rundek T, Sacco RL. Prognosis after stroke. In: Mohr JP, Wolf PhA, Grotta JC, Moskowitz MA, Mayberg MR, von Kummer R (eds). Stroke. Pathophysiology, diagnosis and management (fifth edition). Elsevier, Philadephia, USA, 2011, pp 219-241.

6. Röden-Jüllig A. Progressive stroke: epidemiology. Cerebrovasc Dis 1997; 7 (Suppl 5): 2-5.

7. Toni D. Predictors of stroke deterioration. Cerebrovasc Dis 1997; 7 (Suppl 5): 10-3.

8. Dávalos A. Castillo J. Potential mechanisms of worsening. Cerebrovasc Dis 1997; 7 (Suppl 5): 19-24.

9. Alvarez FJ, Segura T, Castellanos M, Leira R, Blanco M, Castillo J, Davalos A, et al. Cerebral hemodynamic reserve and early neurologic deterioration in acute ischemic stroke. J Cereb Blood Flow Metab. 2004;24(11):1267-1271.

10. Arboix A, Cendros V, Besa M, Garcia-Eroles L, Oliveres $\mathrm{M}$, Targa $\mathrm{C}$, Balcells $\mathrm{M}$, et al. Trends in risk factors, stroke subtypes and outcome. Nineteen-year data from the Sagrat Cor Hospital of Barcelona stroke registry. Cerebrovasc Dis. 2008;26(5):509-516. 
11. Arboix A, Alvarez-Sabin L, Soler L for the Cerebrovascular Study Group of the Spanish Society of Neurology. Nomenclatura de las enfermedades vasculares cerebrales. Neurología 1998; 13 (Suppl 1): 1-10.

12. Special report from the National Institute of Neurological Disorders and Stroke. Classification of cerebrovascular diseases III. Stroke. 1990;21(4):637-676.

13. Pujadas Capmany R, Arboix A, Casanas-Munoz R, Anguera-Ferrando N. Specific cardiac disorders in 402 consecutive patients with ischaemic cardioembolic stroke. Int J Cardiol. 2004;95(2-3):129-134.

14. Arboix A, Garcia-Eroles L, Oliveres M, Massons JB, Targa C. Clinical predictors of early embolic recurrence in presumed cardioembolic stroke. Cerebrovasc Dis. 1998;8(6):345-353.

15. Cote R, Hachinski VC, Shurvell BL, Norris JW, Wolfson $\mathrm{C}$. The Canadian Neurological Scale: a preliminary study in acute stroke. Stroke. 1986;17(4):731-737.

16. Bamford JM, Sandercock PA, Warlow CP, Slattery J. Interobserver agreement for the assessment of handicap in stroke patients. Stroke. 1989;20(6):828.

17. Classification and diagnostic criteria for headache disorders, cranial neuralgias and facial pain. Headache Classification Committee of the International Headache Society. Cephalalgia. 1988;8 (Suppl 7):1-96.

18. Teasdale G, Jennett B. Assessment of coma and impaired consciousness. A practical scale. Lancet. 1974;2(7872):81-84.

19. Proposal for revised clinical and electroencephalographic classification of epileptic seizures. From the Commission on Classification and Terminology of the International League Against Epilepsy. Epilepsia. 1981;22(4):489-501.

20. Grupo de Estudio de las Enfermedades Cerebrovasculares de la Sociedad Española de Neurología: manejo del paciente con enfermedad vascular cerebral aguda. Recoendaciones 1992. Edos, 1992. Barcelona, Spain.

21. Arboix A, Alio J. Acute cardioembolic stroke: an update.
Expert Rev Cardiovasc Ther. 2011;9(3):367-379.

22. Serena J, Leira R, Castillo J, Pumar JM, Castellanos M, Davalos A. Neurological deterioration in acute lacunar infarctions: the role of excitatory and inhibitory neurotransmitters. Stroke. 2001;32(5):1154-1161.

23. Davalos A, Cendra E, Teruel J, Martinez M, Genis D. Deteriorating ischemic stroke: risk factors and prognosis. Neurology. 1990;40(12):1865-1869.

24. Camilo O, Goldstein LB. Seizures and epilepsy after ischemic stroke. Stroke. 2004;35(7):1769-1775.

25. Arboix A, Comes E, Garcia-Eroles L, Massons JB, Oliveres M, Balcells M. Prognostic value of very early seizures for in-hospital mortality in atherothrombotic infarction. Eur Neurol. 2003;50(2):78-84.

26. Lynch MW, Rutecki PA, Sutula TP. The effects of seizures on the brain. Curr Opin Neurol. 1996;9(2):97-102.

27. Bladin CF, Alexandrov AV, Bellavance A, Bornstein N, Chambers B, Cote R, Lebrun L, et al. Seizures after stroke: a prospective multicenter study. Arch Neurol. 2000;57(11):1617-1622.

28. Arboix A, Massons J, Oliveres M, Arribas MP, Titus F. Headache in acute cerebrovascular disease: a prospective clinical study in 240 patients. Cephalalgia. 1994;14(1):37-40.

29. Tentschert S, Wimmer R, Greisenegger S, Lang W, Lalouschek W. Headache at stroke onset in 2196 patients with ischemic stroke or transient ischemic attack. Stroke. 2005;36(2):e1-3.

30. Leira R, Davalos A, Aneiros A, Serena J, Pumar JM, Castillo J. Headache as a surrogate marker of the molecular mechanisms implicated in progressing stroke. Cephalalgia. 2002;22(4):303-308.

31. Castillo J, Martinez F, Corredera E, Aldrey JM, Noya M. Amino acid transmitters in patients with headache during the acute phase of cerebrovascular ischemic disease. Stroke. 1995;26(11):2035-2039.

32. Arboix A. Thrombolytic treatment in acute cerebral infarction. Neurol India. 2007;55(1):8-9. 